\title{
Correction to: Categorical Relations Between Langlands Dual Quantum Affine Algebras: Exceptional Cases
}

\author{
Se-jin Oh ${ }^{1}$, Travis Scrimshaw ${ }^{2}$ \\ 1 Ewha Womans University Seoul, 52 Ewhayeodae-gil, Daehyeon-dong, Seodaemun-gu, Seoul, South Korea. \\ E-mail: sejin092@gmail.com; URL: https://sites.google.com/site/mathsejinoh/ \\ 2 School of Mathematics and Physics, The University of Queensland, St. Lucia, QLD 4072, Australia. \\ E-mail: tcscrims@gmail.com; URL: https://people.smp.uq.edu.au/TravisScrimshaw/
}

Received: 10 June 2019 / Accepted: 29 July 2019

Published online: 5 October 2019 - (C) Springer-Verlag GmbH Germany, part of Springer Nature 2019

\author{
Correction to: Commun. Math. Phys. 368, 295-367 (2019) \\ https://doi.org/10.1007/s00220-019-03287-w
}

In this addendum, we remove the ambiguity for roots of higher order of denominator formulas in our paper. These refinements state that there are roots of order 4, 5, 6, which is the first such observation of a root of order strictly larger than 3 to the best knowledge of the authors.

\section{Introduction}

In this short note, we remove the ambiguities in our paper [2, Prop. 4.29, Prop. 7.8, App. C, App. D]. We will use the same notation as in [2]; in particular, $d_{i, j}(z):=d_{i, j}^{X_{n}^{(r)}}(z)$ denotes the denominator formula for $R_{V\left(\varpi_{i}\right)_{x}, V\left(\varpi_{j}\right)_{y}}^{\text {norm }}$ in the affine Lie algebra of type $X_{n}^{(r)}$, where $z=x / y$. We also require the following.

Proposition 1.1 [3, Prop. 6.8]. Let $Q$ be a Dynkin quiver of finite simply laced type. For $a[Q]$-minimal pair $(\alpha, \beta)$ of a simple sequence $\underline{s}=\left(\alpha_{1}, \ldots, \alpha_{k}\right), V_{Q}^{(1)}\left(\alpha_{1}\right) \otimes \cdots \otimes$ $V_{Q}^{(1)}\left(\alpha_{k}\right)$ is simple, and there exists a surjective homomorphism

$$
V_{Q}^{(1)}(\beta) \otimes V_{Q}^{(1)}(\alpha) \rightarrow V_{Q}^{(1)}\left(\alpha_{1}\right) \otimes \cdots \otimes V_{Q}^{(1)}\left(\alpha_{k}\right)
$$

Se-jin Oh was supported by the Ministry of Education of the Republic of Korea and the National Research Foundation of Korea (NRF-2019R1A2C4069647). Travis Scrimshaw was partially supported by the Australian Research Council DP170102648.

The original article can be found online at https://doi.org/10.1007/s00220-019-03287-w. 


\section{Type $E_{6}^{(1)}$ and $E_{6}^{(2)}$}

In [2, Prop. 4.29], there was the ambiguity

$$
d_{4,4}^{E_{6}^{(1)}}(z)=\left(z-q^{2}\right)\left(z-q^{4}\right)^{2}\left(z-q^{6}\right)^{2+\epsilon}\left(z-q^{8}\right)^{3}\left(z-q^{10}\right)^{2}\left(z-q^{12}\right)
$$

for some $\epsilon \in\{0,1\}$. We shall show that $\epsilon=1$.

Proposition 2.1. For type $E_{6}^{(1)}$, we have

$$
d_{4,4}(z)=\left(z-q^{2}\right)\left(z-q^{4}\right)^{2}\left(z-q^{6}\right)^{3}\left(z-q^{8}\right)^{3}\left(z-q^{10}\right)^{2}\left(z-q^{12}\right) .
$$

Proof. Note that, from the AR-quiver of type $E_{6}$ in [2, (2.6)], we can read that

$$
\left(\left(\begin{array}{l}
111 \\
100
\end{array}\right),\left(\begin{array}{l}
101 \\
110
\end{array}\right)\right) \text { is a }[Q] \text {-minimal pair of the simple sequence }\left(\left(\begin{array}{l}
101 \\
100
\end{array}\right),\left(\begin{array}{l}
111 \\
110
\end{array}\right)\right) \text {. }
$$

Hence Proposition 1.1 states that we have a homomorphism

$$
V\left(\varpi_{1}\right)_{(-q)^{-3}} \otimes V\left(\varpi_{4}\right)_{(-q)^{1}} \rightarrow V\left(\varpi_{2}\right) \otimes V\left(\varpi_{5}\right) .
$$

Then we have

$$
\frac{d_{1,4}\left((-q)^{-3} z\right) d_{4,4}\left((-q)^{1} z\right)}{d_{2,4}(z) d_{5,4}(z)} \times \frac{a_{2,4}(z) d_{5,4}(z)}{a_{1,4}\left((-q)^{-3} z\right) a_{4,4}\left((-q)^{1} z\right)} \in \mathbf{k}\left[z^{ \pm 1}\right]
$$

by [1, Lemma C.15]. From [2, Lemma 3.9], one can compute that

$$
\begin{aligned}
a_{2,4}(z) & \equiv \frac{[19][5][23][1]}{[7][17][11][13]} & a_{3,4}(z) & \equiv a_{4,5}(z) \equiv \frac{[1][3][21][23]}{[9][11][13][15]} \\
a_{1,4}(z) & \equiv \frac{[2][22]}{[10][14]} & a_{4,4}(z) & \equiv \frac{[0][2][4][20][22][24]}{[8][10][12]^{2}[14][16]} .
\end{aligned}
$$

Since we have computed $d_{1,4}(z), d_{2,4}(z)$ and $d_{5,4}(z),(2.3)$ can be written as follows:

$$
\frac{d_{1,4}\left((-q)^{-3} z\right) d_{4,4}\left((-q)^{1} z\right)}{d_{2,4}(z) d_{5,4}(z)}=\frac{\left(z+q^{1}\right)\left(z+q^{3}\right)^{2}\left(z+q^{5}\right)^{2} \text { or } 3\left(z+q^{7}\right)^{4}\left(z-q^{9}\right)^{3}\left(z-q^{11}\right)^{2}\left(z+q^{13}\right)}{\left(z+q^{3}\right)^{2}\left(z+q^{5}\right)^{3}\left(z+q^{7}\right)^{4}\left(z+q^{9}\right)^{3}\left(z+q^{11}\right)^{2}}
$$

by (2.1) and

$$
\begin{aligned}
\frac{a_{2,4}(z) d_{5,4}(z)}{a_{1,4}\left((-q)^{-3} z\right) a_{4,4}\left((-q)^{1} z\right)} & =\frac{[19][5][23][1]}{[7][1]][11][13]} \times \frac{[1][3][21][23]}{[9][11][13][15]} \times \frac{[7][11]}{[-1][19]} \times \frac{[9][11][13]^{2}[15][17]}{[1][3][5][21][23][25]} \\
& =\frac{[23][1]}{[-1][25]}=\frac{\left(z-(-q)^{-1}\right)}{\left(z-(-q)^{1}\right)} .
\end{aligned}
$$

Thus we have

$$
\begin{aligned}
& \frac{\left(z+q^{1}\right)\left(z+q^{3}\right)^{2}\left(z+q^{5}\right)^{2 \text { or } 3}\left(z+q^{7}\right)^{4}\left(z-q^{9}\right)^{3}\left(z-q^{11}\right)^{2}\left(z+q^{13}\right)}{\left(z+q^{3}\right)^{2}\left(z+q^{5}\right)^{3}\left(z+q^{7}\right)^{4}\left(z+q^{9}\right)^{3}\left(z+q^{11}\right)^{2}} \times \frac{\left(z-(-q)^{-1}\right)}{\left(z-(-q)^{1}\right)} \\
& =\frac{\left(z+q^{-1}\right)\left(z+q^{3}\right)^{2}\left(z+q^{5}\right)^{2} \text { or } 3\left(z+q^{7}\right)^{4}\left(z-q^{9}\right)^{3}\left(z-q^{11}\right)^{2}\left(z+q^{13}\right)}{\left(z+q^{3}\right)^{2}\left(z+q^{5}\right)^{3}\left(z+q^{7}\right)^{4}\left(z+q^{9}\right)^{3}\left(z+q^{11}\right)^{2}} \in \mathbf{k}\left[z^{ \pm 1}\right]
\end{aligned}
$$

which implies that the order of degree $(-q)^{6}$ should be 3 . 
By applying generalized Schur-Weyl duality

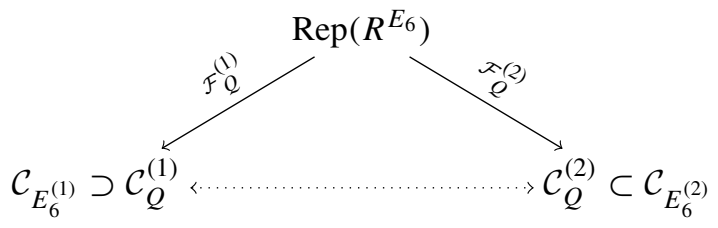

we have the twisted analogue of Proposition 1.1:

Proposition 2.2. Let $Q$ be a Dynkin quiver offinite simply-laced type. For a $[Q]$-minimal pair $(\alpha, \beta)$ of a simple sequence $\underline{s}=\left(\alpha_{1}, \ldots, \alpha_{k}\right), V_{Q}^{(2)}\left(\alpha_{1}\right) \otimes \cdots \otimes V_{Q}^{(2)}\left(\alpha_{k}\right)$ is simple, and there exists a surjective homomorphism

$$
V_{Q}^{(2)}(\beta) \otimes V_{Q}^{(2)}(\alpha) \rightarrow V_{Q}^{(2)}\left(\alpha_{1}\right) \otimes \cdots \otimes V_{Q}^{(2)}\left(\alpha_{k}\right)
$$

In particular, (2.2) transfers to the following homomorphism in $\mathcal{C}_{Q}^{(2)}$ under the map in [2, Prop. 6.5]:

$$
V\left(\varpi_{1}\right)_{(-q)^{-3}} \otimes V\left(\varpi_{3}\right)_{\sqrt{-1}(-q)^{1}} \rightarrow V\left(\varpi_{2}\right)_{-1} \otimes V\left(\varpi_{4}\right)_{\sqrt{-1}} .
$$

Next, we resolve from [2, Prop. 7.8] the ambiguity in type $E_{6}^{(2)}$

$$
d_{3,3}^{E_{6}^{(2)}}(z)=\left(z^{2}-q^{4}\right)\left(z^{2}-q^{8}\right)^{2}\left(z^{2}-q^{12}\right)^{2+\epsilon^{\prime}}\left(z^{2}-q^{16}\right)^{3}\left(z^{2}-q^{20}\right)^{2}\left(z^{2}-q^{24}\right)
$$

for some $\epsilon^{\prime} \in\{0,1\}$ by showing $\epsilon^{\prime}=1$.

Proposition 2.3. For type $E_{6}^{(2)}$, we have

$$
d_{3,3}(z)=\left(z^{2}-q^{4}\right)\left(z^{2}-q^{8}\right)^{2}\left(z^{2}-q^{12}\right)^{3}\left(z^{2}-q^{16}\right)^{3}\left(z^{2}-q^{20}\right)^{2}\left(z^{2}-q^{24}\right) .
$$

Proof. By (2.4) and [1, Lemma C.15], we have

$$
\frac{d_{1,3}\left(\sqrt{-1}(-q)^{-3} z\right) d_{3,3}\left((-q)^{1} z\right)}{d_{2,3}(\sqrt{-1} z) d_{3,4}(z)} \times \frac{a_{2,3}(\sqrt{-1} z) a_{3,4}(z)}{a_{1,3}\left(\sqrt{-1}(-q)^{-3} z\right) a_{3,3}\left((-q)^{1} z\right)} \in \mathbf{k}\left[z^{ \pm 1}\right] .
$$

From [2, Lemma 3.9], one can compute that

$$
\begin{array}{lll}
a_{2,3}(\sqrt{-1} z) \equiv \frac{\{3\}\{21\}\{1\}\{23\}}{\{9\}\{15\}\{11\}\{13\}}, & a_{3,4}(z) \equiv \frac{\{5\}\{19\}\{1\}\{23\}}{\{7\}\{17\}\{11\}\{13\}}, \\
a_{1,3}(\sqrt{-1} z) \equiv \frac{\{2\}\{22\}}{\{10\}\{14\}}, & a_{3,3}(z) \equiv \frac{\{4\}\{20\}\{2\}\{22\}\{0\}\{24\}}{\{8\}\{16\}\{10\}\{14\}\{12\}^{2}} .
\end{array}
$$

Since we have computed $d_{1,3}(z), d_{2,3}(z)$ and $d_{3,4}(z),(2.6)$ can be written as follows:

$$
\begin{aligned}
& \frac{d_{1,3}\left(\sqrt{-1}(-q)^{-3} z\right) d_{3,3}\left((-q)^{1} z\right)}{d_{2,3}(\sqrt{-1} z) d_{3,4}(z)} \\
& =\frac{\left(z^{2}-q^{2}\right)\left(z^{2}-q^{6}\right)^{2}\left(z^{2}-q^{10}\right)^{2} \text { or } 3\left(z^{2}-q^{14}\right)^{4}\left(z^{2}-q^{18}\right)^{3}\left(z^{2}-q^{22}\right)^{2}\left(z^{2}-q^{26}\right)}{\left(z^{2}-q^{6}\right)^{2}\left(z^{2}-q^{10}\right)^{3}\left(z^{2}-q^{14}\right)^{4}\left(z^{2}-q^{18}\right)^{3}\left(z^{2}-q^{22}\right)^{2}}
\end{aligned}
$$


by $(2.5)$ and

$$
\begin{aligned}
& \frac{a_{2,3}(\sqrt{-1} z) a_{3,4}(z)}{a_{1,3}\left(\sqrt{-1}(-q)^{-3} z\right) a_{3,3}\left((-q)^{1} z\right)} \\
& =\frac{\{3\}\{21\}\{1\}\{23\}}{\{9\}\{15\}\{11\}\{13\}} \times \frac{\{5\}\{19\}\{1\}\{23\}}{\{7\}\{17\}\{11\}\{13\}} \times \frac{\{7\}\{11\}}{\{-1\}\{19\}} \times \frac{\{9\}\{17\}\{11\}\{15\}\{13\}^{2}}{\{5\}\{21\}\{3\}\{23\}\{1\}\{25\}} \\
& =\frac{\{1\}\{23\}}{\{25\}\{-1\}}=\frac{\left(z^{2}-q^{-2}\right)}{\left(z^{2}-q^{2}\right)} .
\end{aligned}
$$

Thus we have

$$
\frac{\left(z^{2}-q^{-2}\right)\left(z^{2}-q^{6}\right)^{2}\left(z^{2}-q^{10}\right)^{2} \text { or } 3\left(z^{2}-q^{14}\right)^{4}\left(z^{2}-q^{18}\right)^{3}\left(z^{2}-q^{22}\right)^{2}\left(z^{2}-q^{26}\right)}{\left(z^{2}-q^{6}\right)^{2}\left(z^{2}-q^{10}\right)^{3}\left(z^{2}-q^{14}\right)^{4}\left(z^{2}-q^{18}\right)^{3}\left(z^{2}-q^{22}\right)^{2}} \in \mathbf{k}\left[z^{ \pm 1}\right],
$$

which implies our assertion.

\section{Types $E_{7}^{(1)}$ and $E_{8}^{(1)}$}

In [2, App. C,D], there were several denominators which contain ambiguity for roots of higher order (note that there were several typos in [2, App. C,D]):

$$
\begin{aligned}
& d_{3,4}^{E_{7}^{(1)}}(z)=\left(z+q^{3}\right)\left(z+q^{5}\right)^{2}\left(z+q^{7}\right)^{2}\left(z+q^{9}\right)^{2+\epsilon_{1}^{(1)}}\left(z+q^{11}\right)^{3}\left(z+q^{13}\right)^{2}\left(z+q^{15}\right)^{2}\left(z+q^{17}\right), \\
& d_{4,5}^{E_{7}^{(1)}}(z)=\left(z+q^{3}\right)\left(z+q^{5}\right)^{2}\left(z+q^{7}\right)^{2+\epsilon_{1}^{(1)}}\left(z+q^{9}\right)^{2+\epsilon_{1}^{(2)}}\left(z+q^{11}\right)^{2+\epsilon}\left(z+q^{13}\right)^{3}\left(z+q^{15}\right)^{2}\left(z+q^{17}\right) \text {, } \\
& d_{4,4}^{E_{7}^{(1)}}(z)=\left(z-q^{2}\right)\left(z-q^{4}\right)^{2}\left(z-q^{6}\right)^{2+\epsilon_{1}^{(1)}}\left(z-q^{8}\right)^{2+\epsilon_{2}^{(1)}} \\
& \times\left(z-q^{10}\right)^{2+\epsilon_{2}^{(1)}}\left(z-q^{12}\right)^{3+\epsilon_{1}^{(1)}}\left(z-q^{14}\right)^{3}\left(z-q^{16}\right)^{2}\left(z-q^{18}\right), \\
& d_{2,4}^{E_{8}^{(1)}}(z)=\left(z+q^{3}\right)\left(z+q^{5}\right)\left(z+q^{7}\right)^{2}\left(z+q^{9}\right)^{2}\left(z+q^{11}\right)^{2+\epsilon_{1}^{(1)}}\left(z+q^{13}\right)^{2+\epsilon_{1}^{(2)}}\left(z+q^{15}\right)^{2+\epsilon_{1}^{(3)}} \\
& \times\left(z+q^{17}\right)^{2+\epsilon_{1}^{(2)}}\left(z+q^{19}\right)^{2+\epsilon_{1}^{(1)}}\left(z+q^{21}\right)^{3}\left(z+q^{23}\right)^{2}\left(z+q^{25}\right)^{2}\left(z+q^{27}\right)\left(z+q^{29}\right), \\
& d_{3,3}^{E_{8}^{(1)}}(z)=\left(z-q^{2}\right)\left(z-q^{4}\right)\left(z-q^{6}\right)\left(z-q^{8}\right)^{2}\left(z-q^{10}\right)^{2}\left(z-q^{12}\right)^{2+\epsilon_{1}^{(1)}}\left(z-q^{14}\right)^{3}\left(z-q^{16}\right)^{2} \\
& \times\left(z-q^{18}\right)^{2+\epsilon_{1}^{(1)}}\left(z-q^{20}\right)^{3}\left(z-q^{22}\right)^{2}\left(z-q^{24}\right)^{2}\left(z-q^{26}\right)\left(z-q^{28}\right)\left(z-q^{30}\right), \\
& d_{3,4}^{E_{8}^{(1)}}(z)=\left(z+q^{3}\right)\left(z+q^{5}\right)^{2}\left(z+q^{7}\right)^{2}\left(z+q^{9}\right)^{2+\epsilon_{1}^{(1)}}\left(z+q^{11}\right)^{2+\epsilon_{2}^{(1)}}\left(z+q^{13}\right)^{2+\epsilon_{2}^{(2)}}\left(z+q^{15}\right)^{2+\epsilon_{2}^{(3)}} \\
& \times\left(z+q^{17}\right)^{2+\epsilon_{2}^{(2)}}\left(z+q^{19}\right)^{2+\epsilon_{2}^{(1)}}\left(z+q^{21}\right)^{3+\epsilon_{1}^{(1)}}\left(z+q^{23}\right)^{3}\left(z+q^{25}\right)^{2}\left(z+q^{27}\right)^{2}\left(z+q^{29}\right), \\
& d_{3,5}^{E_{8}^{(1)}}(z)=\left(z-q^{4}\right)\left(z-q^{6}\right)^{2}\left(z-q^{8}\right)^{2}\left(z-q^{10}\right)^{2+\epsilon_{1}^{(1)}}\left(z-q^{12}\right)^{2+\epsilon_{1}^{(2)}}\left(z-q^{14}\right)^{2+\epsilon_{1}^{(3)}}\left(z-q^{16}\right)^{3+\epsilon_{1}^{(3)}} \\
& \times\left(z-q^{18}\right)^{2+\epsilon_{1}^{(2)}}\left(z-q^{20}\right)^{2+\epsilon_{1}^{(1)}}\left(z-q^{22}\right)^{3}\left(z-q^{24}\right)^{2}\left(z-q^{26}\right)^{2}\left(z-q^{28}\right), \\
& d_{3,6}^{E_{8}^{(1)}}(z)=\left(z+q^{5}\right)\left(z+q^{7}\right)^{2}\left(z+q^{9}\right)^{2}\left(z+q^{11}\right)^{2}\left(z+q^{13}\right)^{2}\left(z+q^{15}\right)^{2+\epsilon_{1}^{(1)}}\left(z+q^{17}\right)^{3} \\
& \times\left(z+q^{19}\right)^{2}\left(z+q^{21}\right)^{2}\left(z+q^{23}\right)^{2}\left(z+q^{25}\right)^{2}\left(z+q^{27}\right), \\
& d_{4,4}^{E_{8}^{(1)}}(z)=\left(z-q^{2}\right)\left(z-q^{4}\right)^{2}\left(z-q^{6}\right)^{2+\epsilon_{1}^{(1)}}\left(z-q^{8}\right)^{2+\epsilon_{2}^{(1)}}\left(z-q^{10}\right)^{2+\epsilon_{3}^{(1)}}\left(z-q^{12}\right)^{2+\epsilon_{4}^{(1)}}\left(z-q^{14}\right)^{2+\epsilon_{4}^{(2)}} \\
& \times\left(z-q^{16}\right)^{2+\epsilon_{4}^{(2)}}\left(z-q^{18}\right)^{2+\epsilon_{4}^{(1)}}\left(z-q^{20}\right)^{3+\epsilon_{3}^{(1)}}\left(z-q^{22}\right)^{3+\epsilon_{2}^{(1)}}\left(z-q^{24}\right)^{3+\epsilon_{1}^{(1)}}
\end{aligned}
$$


Table 1. The minimal pairs $\underline{p}$ and their $\operatorname{socles} \operatorname{soc}_{Q}(\underline{p})$ of $\Gamma_{Q}$ in [2, App. A]

\begin{tabular}{llll}
\hline Minimal pair $\underline{p}$ & $\left(\left(\begin{array}{c}111 \\
110\end{array}\right),\left(\begin{array}{c}1223 \\
221\end{array}\right)\right)$ & $\left(\left(\begin{array}{c}1122 \\
110\end{array}\right),\left(\begin{array}{c}1223 \\
221\end{array}\right)\right)$ & $\left(\left(\begin{array}{c}1224 \\
321\end{array}\right),\left(\begin{array}{c}1011 \\
111\end{array}\right)\right)$ \\
\hline $\operatorname{soc}_{Q}(\underline{p})$ & $\left(\left(\begin{array}{c}0111 \\
110\end{array}\right),\left(\begin{array}{c}1112 \\
110\end{array}\right),\left(\begin{array}{c}1111 \\
111\end{array}\right)\right)$ & $\left(\left(\begin{array}{c}0111 \\
110\end{array}\right),\left(\begin{array}{c}1112 \\
110\end{array}\right),\left(\begin{array}{c}1122 \\
111\end{array}\right)\right)$ & $\left(\left(\begin{array}{c}1112 \\
211\end{array}\right),\left(\begin{array}{c}1123 \\
221\end{array}\right)\right)$ \\
\hline
\end{tabular}

$$
\begin{aligned}
& \times\left(z-q^{26}\right)^{3}\left(z-q^{28}\right)^{2}\left(z-q^{30}\right), \\
d_{4,5}^{E_{8}^{(1)}}(z)= & \left(z+q^{3}\right)\left(z+q^{5}\right)^{2}\left(z+q^{7}\right)^{2+\epsilon_{1}^{(1)}}\left(z+q^{9}\right)^{2+\epsilon_{2}^{(1)}}\left(z+q^{11}\right)^{2+\epsilon_{2}^{(2)}}\left(z+q^{13}\right)^{2+\epsilon_{3}^{(1)}}\left(z+q^{15}\right)^{2+\epsilon_{3}^{(2)}} \\
& \times\left(z+q^{17}\right)^{2+\epsilon_{3}^{(1)}}\left(z+q^{19}\right)^{3+\epsilon_{2}^{(2)}}\left(z+q^{21}\right)^{2+\epsilon_{2}^{(1)}}\left(z+q^{23}\right)^{3+\epsilon_{1}^{(1)}}\left(z+q^{25}\right)^{3}\left(z+q^{27}\right)^{2}\left(z+q^{29}\right), \\
d_{4,6}^{E_{8}^{(1)}}(z)= & \left(z-q^{4}\right)\left(z-q^{6}\right)^{2}\left(z-q^{8}\right)^{2+\epsilon_{1}^{(1)}}\left(z-q^{10}\right)^{2+\epsilon_{1}^{(2)}}\left(z-q^{12}\right)^{2+\epsilon_{1}^{(3)}}\left(z-q^{14}\right)^{2+\epsilon_{2}^{(1)}} \\
& \times\left(z-q^{16}\right)^{2+\epsilon_{2}^{(1)}}\left(z-q^{18}\right)^{3+\epsilon_{1}^{(3)}}\left(z-q^{20}\right)^{2+\epsilon_{1}^{(2)}}\left(z-q^{22}\right)^{2+\epsilon_{1}^{(1)}}\left(z-q^{24}\right)^{3}\left(z-q^{26}\right)^{2}\left(z-q^{28}\right), \\
d_{5,5}^{E_{8}^{(1)}}(z)= & \left(z-q^{2}\right)\left(z-q^{4}\right)\left(z-q^{6}\right)^{2}\left(z-q^{8}\right)^{2+\epsilon_{1}^{(1)}}\left(z-q^{10}\right)^{2+\epsilon_{1}^{(2)}}\left(z-q^{12}\right)^{2+\epsilon_{2}^{(1)}}\left(z-q^{14}\right)^{2+\epsilon_{2}^{(2)}} \\
& \times\left(z-q^{16}\right)^{2+\epsilon_{2}^{(2)}}\left(z-q^{18}\right)^{2+\epsilon_{2}^{(1)}}\left(z-q^{20}\right)^{3+\epsilon_{1}^{(3)}}\left(z-q^{22}\right)^{2+\epsilon_{1}^{(1)}}\left(z-q^{24}\right)^{3}\left(z-q^{26}\right)^{2}\left(z-q^{28}\right), \\
d_{5,6}^{E_{8}^{(1)}(z)=} & \left(z+q^{3}\right)\left(z+q^{5}\right)\left(z+q^{7}\right)^{2}\left(z+q^{9}\right)^{2}\left(z+q^{11}\right)^{2+\epsilon_{1}^{(1)}}\left(z+q^{13}\right)^{2+\epsilon_{1}^{(2)}}\left(z+q^{15}\right)^{2+\epsilon_{1}^{(3)}}\left(z+q^{17}\right)^{2+\epsilon_{1}^{(2)}} \\
& \times\left(z+q^{19}\right)^{2+\epsilon_{1}^{(1)}}\left(z+q^{21}\right)^{3}\left(z+q^{23}\right)^{2}\left(z+q^{25}\right)^{2}\left(z+q^{27}\right)\left(z+q^{29}\right),
\end{aligned}
$$

for some $\epsilon_{1}^{(i)} \in\{0,1\}, \epsilon_{2}^{(i)} \in\{0,1,2\}, \epsilon_{3}^{(i)} \in\{0,1,2,3\}$ and $\epsilon_{4}^{(i)} \in\{0,1,2,3,4\}$ $\left(i \in \mathbb{Z}_{\geq 0}\right)\left(i \in \mathbb{Z}_{\geq 0}\right)$.

Applying the same arguments in Sect. 2, we can prove that $\epsilon_{k}^{(i)}=k$ for all $1 \leq k \leq 4$ and $i$. Hence there are roots of order 4, 5, 6 also. To the best knowledge of the authors, this is the first such observation of a root of order strictly larger than 3 .

When we prove for $E_{7}^{(1)}$ cases, we employ the following homomorphisms

$$
\begin{aligned}
& V(3)_{(-q)^{-1}} \otimes V(6)_{(-q)^{4}} \rightarrow V(1) \otimes V(2)_{(-q)} \otimes V(7)_{(-q)^{3}}, \\
& V(3)_{(-q)^{-1}} \otimes V(5)_{(-q)^{3}} \rightarrow V(1) \otimes V(2)_{(-q)} \otimes V(6)_{(-q)^{2},} \\
& V(1)_{(-q)^{-3}} \otimes V(4)_{(-q)^{1}} \rightarrow V(2) \otimes V(5),
\end{aligned}
$$

which can be obtained from Proposition 1.1 and Table 1.

Publisher's Note Springer Nature remains neutral with regard to jurisdictional claims in published maps and institutional affiliations.

\section{References}

1. Akasaka, T., Kashiwara, M.: Finite-dimensional representations of quantum affine algebras. Publ. RIMS. Kyoto Univ. 33, 839-867 (1997)

2. Oh, S.-J., Scrimshaw, T.: Categorical relations between Langlands dual quantum affine algebras: exceptional cases. Commun. Math. Phys. 368(1), 295-367 (2019)

3. Oh, S.-J.: Auslander-Reiten quiver and representation theories related to KLR-type Schur-Weyl duality. Math. Z. 1-2, 499-554 (2019) 